\title{
Bracket-Languages are Recognizable \\ in Logarithmic Space
}

Kurt Meh1horn

Fachbere1ch 10

Angewandte Mathematik und

Informatik der Universitat

des Saarlandes

D-6600 Saarbrücken

Im Stadtwald

A $75 / 12$

September 1975 


\section{Summary:}

In this paper, we prove lower bounds for the space requirement of the membershtpproblem of context-free languages. A contextfree language $I \subseteq \sum^{*} 1 s$ called strongly non-regular if there exilts words $u, v, w, w_{0} y \in \sum^{*}$ with $L \cap u v^{*} w x^{*} y$ non-regular.

Main Theorem: To decide the membershipproblem of strongly nonregular context-free languages requires $O(\log n)$ space infinitely often.

As coroliarles we obtain lower bounds for several familles of determinist1c languages, e.g. realtime languages, and about the minlmal growth rate of tape constructable functions. 
Context-free languages are an important topic in practical as well as in theoretical computer science. Much effort was devoted to the construction of time - and (or) space - efficient recognition algorithms.

Early results were obtalned by Lewis, Hartmanis and Stearns [5] : Every context-free language can be recognized on an off-ilne Turing machine in space $O\left(\log ^{2} n\right)$, where $n$ is the length of the Input. Recent results by Sudborough [7] and Monien [6] indicate that it is probably very hard to beat this bound. They showed that the existence of a general context-free recognition algorithm working in logarithmic space would imply the equality of deterministic of nondeterministic context-sensitive languages, $1 . e$. a solution to Myhill's LBA-problem.

However, it is conceivable that large subclasses of the contextfree languages (e.g. the deterministic languages) are recognizable in less than $O\left(\log ^{2} n\right)$ units of space. In [1] Alt and Mehlhorn attacked this problem by proving that the word-problem for many classes of deterministic context-free languages requires at least $O(\log n)$ units of space infinttely often. Hotz and Messerschmidt [4] attacked the problem from the other end: Dyck-languages can be recognized in $O(\log n)$ units of space. We extend their results and show that every bracket-language can be recognized in $O(\log n)$ units of space.

For the notation we refer the reader to [3]. A context-free gramar is a 4-tuple $G=(V,[, P, S)$, where $V$ is the set of non-terminals (variables), [1s the set of terminals, $P$ is the set of productions and $S$ is the startsymbol. $($,$) are two special symbols in [$. A production $A \rightarrow(\alpha)$ is called bracketed iff $\alpha \in\left(([\cup V)-\{(,)\})^{*}\right.$. A context-free language $I$ is a bracket-language if it can be generated by a context-free grammax $G$ all of whlch rules are bracketed. The bracketing is a linear encoding of the syntax tree. In the sequel, I 18 always a bracket-language and $w \in \sum^{*}$ is a word of length $n$. We want to declde if $w \in I$. 
First, we find out if there is a correct number of brackets in w. To do so, we scan $w$ from left to right once always keeping a count (in binary) of the number of unmatched open brackets. This number should always be positive except when scanning the very last symbol of $w$.

If $w$ falls this test then it is rejected. Otherwise, the bxackets in $w$ encode a tree. This tree must be the parse-tree. So, we only have to find out if there is a consistent labeling of the interior nodes of the tree by the variables of the grammar. This can be done by labelling the tree bottom-up according to the following rules:

I) The leaves of the tree are labelled by the symbols of w from left to right.

II) Let $v_{0}$ be a node with sons $v_{1} \ldots, v_{k}$. If $v_{1}$ is labelled by $v_{1} \leq v_{1} l \leq 1 \leq k$, then $v_{0}$ can be labelled by $V_{0}=\left\{A: A+A_{1} A_{2} \ldots A_{k} \in P\right.$ and $A_{1} \in V_{1}$ for $\left.1 \leq 1 \leq k\right\}$

A tree is a parse of $w$ according to $G$ if the label of the root contains the start symbol $\mathrm{s}$. Note the similarity of this labeling procedure with Younger's recognition algorithm [8].

The label of a node is used only once: when its father is labelled. Thereafter its label is never used again. Therefore we can discard it.

Definition:An interior node (non-leaf) $v$ is called essential if it is labelled, but its father is not labelled.

We want to label the rodes of the tree in such a fudiclous order, so to keep the maximal number of nodes which are simultaneously essentlal as small as possible. There is a well known strategy 
for achleving this: Label larger subtrees first $[2, \overline{5}]$.

It is incorporated in the following marking procedure.

procedure label (tree T) :

begin let $v$ be the root of $T$;

1 all of $\mathrm{v}^{\prime} \mathrm{s}$ sons are leaves

then

begin

let the 1 -th son of $v$ be labelled

by $a_{1} \in[, 1 \leq 1 \leq k$;

label $v$ with $\left\{A ; A \rightarrow a_{1} \ldots a_{k} \in P\right\}$;

comment $v$ is now an essential node;

end

else

begin

let $\mathrm{T}_{1}, \mathrm{~T}_{2}, \ldots, \mathrm{T}_{\mathrm{k}}$ be the direct subtrees of $\mathrm{T}$;

let $\pi$ be that permutation of $\{1, \ldots, k\}$

satisfying

(1) ||$T_{\Pi(1)}|| \geq|| T_{\Pi(2)}|| \geq \ldots \geq|| T_{\Pi(k)}||$

(2) ||$T_{\Pi(z)}||=|| T_{\Pi(z+1)} \mid$ implies $\Pi(z)<\Pi(z+1)$

(Here || $\mathrm{T}||$ is the number of leaves of tree $\mathrm{T}$ )

for $l=1$ to $k$ do label (T);

let $v_{1}, 1 \leq 1 \leq k$, be the label of the root

of subtree $\mathrm{T}_{1}$;

label $\mathrm{v}$ by

$\left\{A ; A \rightarrow A_{1} \ldots A_{k} \in P\right.$ and $\left.A_{1} \in V_{1}\right\}$

commont $v$ is now an essential node,

the roots of the subtrees $\mathrm{I}_{1}$ are not esent1al anymore:

end;

end: 
The following facts are easily proved by induction on the data structure.

Fact 1: $([3])$ At most $r \cdot(\log |w|+1)$ nodes are simultaneously essential, where $r=\max \{|\alpha| ; A+\alpha \in P\}$.

Consider any call of the recursive marking procedure. Let $T$ ' be the argument of the active call and let $v^{\prime}$ be 1 ts root. Let further $v_{m}, v_{m-1}, \ldots, v_{1}$ with $v^{\prime}=v_{m}$ be the path from $v^{\prime}$ to the root.

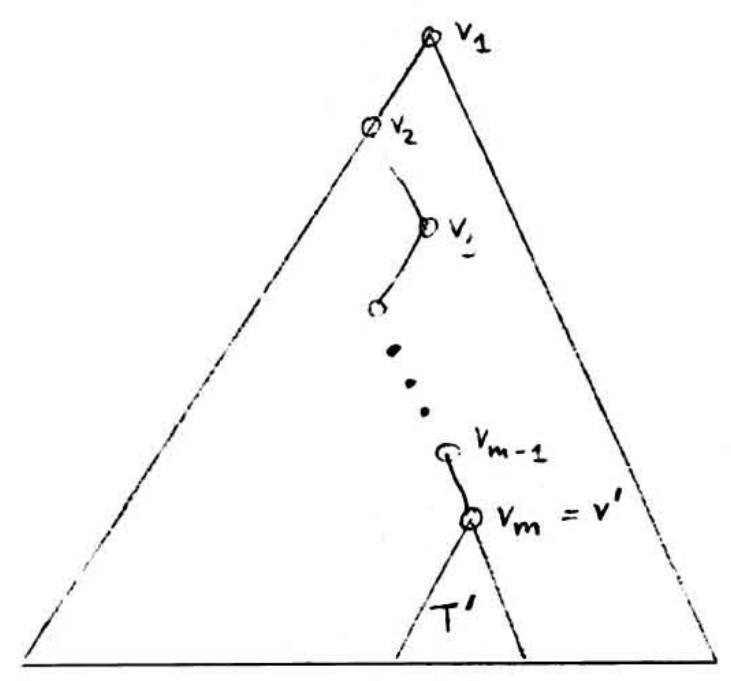

Fact 2: Let $v$ be any essential node. Then $v$ is the son of some $v_{1}$ for $1 \leq 1 \leq \mathrm{m}-1$.

Fact 3: If none of $v_{1}$ 's sons ( $\left.1 \leq 1 \leq m-1\right)$ is essential then $v_{1+1}$ is the root of the leftmost subtree (of the tree with root $v_{i}$ ) having a maximal number of leaves.

We may summarize these three facts as follows: The essential information is concentrated along the path from the root of the argument of the active call to the root of the total tree. The amount of essential information is logarithmically bounded in the length of the input. 
Definltion: A node $v$ is called partializ analyzed if at least one, but not all of 1 ts sons are labelled.

Note that the partlally analyzed nodes are exactly the fathers of essential nodes. Therefore we may reformulate the three facts listed above.

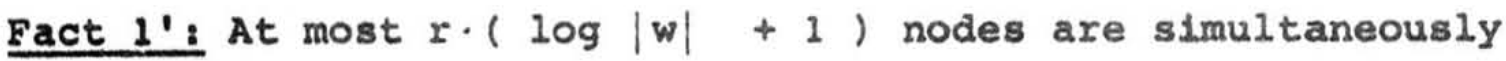
partially analyzed.

Fact 2': Let $v$ be any partially analyzed node. Then $v$ is some $v_{1}, 1 \leq 1 \leq \mathrm{m}-1$.

Fact $3^{\prime}$ : If $v_{1}, 1 \leq 1 \leq m-1$, is not partially analyzed, then $v_{1+1}$ is the root of the leftmost subtree (of the tree with root $v_{1}$ ) having a maximal number of leaves.

We associate with every partlally analyzed node an r-tuple over $2^{\mathrm{v}} \cup\{*\}$. The $z$-th component of this $r$-tuple is

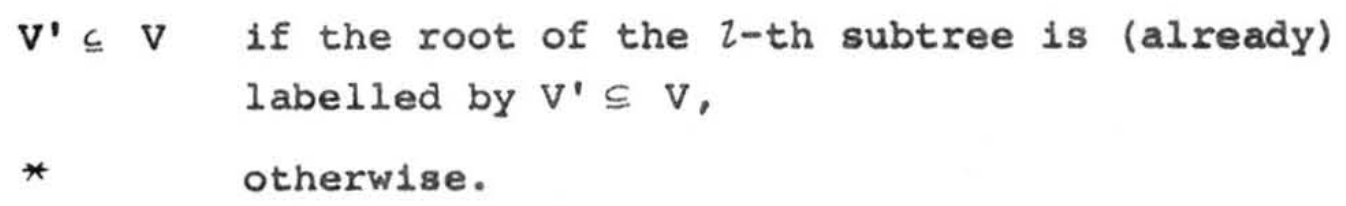

Using these concepts we can rewrite our algorithm. We keep the r-tuples assigned to the partially analyzed nodes in a stack. As before, we label larger subtrees first. Whenever we return from a call of label, with argument $T$ say, we recompute the rank (leftmost largest, rightmost smallest, other) of the argument tree among its brother trees. If $T$ is the leftmost largest subtree then we create a new $r$-tuple and push it on the stack. Then we proceed with the second largest subtree. If $T$ is the rightmost smallest subtree then all 1ts brother trees are already labelled and their labels are stored in the top element of the stack. We use this element to compute the label of the root of the supertree of $T$ and pop the stack. Then we return one level in the recursion. Otherwise we update the top stack element and proceed with the next smaller subtree. This leads to the following non-recursive program. 
(1) tree: $\mathrm{T}$; subset of $\mathrm{V}: \mathrm{H}$;

(2) let $\mathrm{T}$ have $\mathrm{k}$ subtrees :

(3) 1f $k>r$ then reject ;

(4) If all of T's subtrees are leaves

(5) then begin let $a_{1}$ be the label of the 1-th subtree;

(6) $\mathrm{H} \leftarrow\left\{A ; A \rightarrow a_{1} \ldots a_{k} \in P\right\}$

end

(7) else begin $T-$ leftmost maximal subtree of $T$;

(8) goto (4)

end

(9) compute the position of $T$ among its brother trees;

(10) case position of

(11) leftmost largest: create a new r-tuple;

(12) If $T$ is the $Z$-th subtree then let its

l-th component be $\mathrm{H}$;

(13)

push the $\mathbf{r}$-tuple on the stack;

(14) rightmost smallest: if $T$ is the $l$-th subtree then set the l-th component of the top stack element to H:

(15)

let $k$ be the number of non * components of the stack top: $H \leftarrow\left\{A ; A \rightarrow A_{1} \ldots A_{k} \in P\right.$ and $A_{l} \in l-t h$ component of stack top\}; pop the stack;

$\mathrm{T} \leftarrow$ super-tree of $\mathrm{T}$; goto (9) 
(20) other : If $T$ is the 2 -th subtree then set the l-th component of the stack-top to $\mathrm{H}$;

\section{esac:}

(21) T\& next smaller brother of $T$;

(22) goto (2):

The correctness of this algorithm follows from the preceding discussion. Furthermore, fact 1 ensures us that the length of the stack will never exceed.r $(\log |w|+1)$. It is obvious that lines (2), (3), (7), (9), (18) and (21) can be computed in logarithmic space. Therefore we obtain.

Theorem: Every bracket language can be recognized by a $O(\log n)$ space-bounded Turing machine.

Alt and Mehlhorn show in [1] that bracket languages also require $O(\log n)$ space infinitely often.

Corollary: The space complexity of the word problem for bracket languages is exactly $O(\log n)$.

We want to close with a remark on the time complexity of our recognition procedure. Since the machine is $O(\log n)$ spacebounded it is certainly polynomially time bounded. In fact, it is easily seen that the time complexity is $O\left(n^{2}\right)$. 


\section{Bibllography}

[1] H. Alt, K. Mehlhorn : Lower Bounds for the Space Requirement of Some Families of Context-Free Languages, Techn. Berlcht der Untv. des Saarlandes 1975.

[2] Cook, S.A.: An Observation on time-storage trade-off, ICSS, 9, 308-316 (1974)

[3] Hopcroft, J.E and Ullman J.D.: Formal languages and their relation to automata, Addison-Wesley, Reading, Mass., 1969

|4| Hotz, G. and Messerschmldt, J.: Dyck-Sprachen sind in Bandkomplexitat $\log n$ analysierbar, Techn. Bericht der Un1v. des Saarlandes, 1974

[5] Lew1s, P.M., Hartman1s, J., and Stearns, R.E.: Memory bounds for the recognition of context-free and context-sensitive languages, IEEE Conference Record on Switching circuit Theory and Logical Design, 191-202, 1965

[6] Monien, B.: Transformational methods and their application to complexity problems, 2-te GI-Fachtagung, Kaiserslautern, 1975

[7] Sudborough, I.H.: On tape-bounded complexity classes and multihead finite automata, 14 th IEEE SWAT Conference, $138-144,1973$

[8] Younger, D.H.: Recognition and parsing of context-free languages in time $\mathrm{n}^{3}$. Inf. and Control, 10, 189-208, 1967 